\title{
Positive Clinical Impact of an Additional PET/CT Scan Before Adjuvant Radiotherapy or Concurrent Chemoradiotherapy in Patients with Advanced Oral Cavity Squamous Cell Carcinoma
}

\author{
Chung-Jan Kang*1, Chien-Yu Lin*2, Lan-Yan Yang ${ }^{3}$, Tsung-Ying Ho ${ }^{4}$, Li-Yu Lee ${ }^{5}$, Kang-Hsing Fan² ${ }^{2}$ Hung-Ming Wang ${ }^{6}$, \\ Shiang-Fu Huang ${ }^{1}$, Kai-Ping Chang ${ }^{1}$, Ku-Hao Fang ${ }^{1}$, Shu-Hang Ng ${ }^{7}$, Chun-Ta Liao ${ }^{\dagger 1}$, and Tzu-Chen Yen ${ }^{\dagger 4}$ \\ ${ }^{I}$ Department of Otorhinolaryngology, Head and Neck Surgery, Chang Gung Memorial Hospital and Chang Gung University, \\ Taoyuan, Taiwan; ${ }^{2}$ Department of Radiation Oncology, Chang Gung Memorial Hospital and Chang Gung University, Taoyuan, \\ Taiwan; ${ }^{3}$ Biostatistics and Informatics Unit, Clinical Trial Center, Chang Gung Memorial Hospital and Chang Gung University, \\ Taoyuan, Taiwan; ${ }^{4}$ Department of Nuclear Medicine and Molecular Imaging Center, Chang Gung Memorial Hospital and Chang \\ Gung University, Taoyuan, Taiwan; ${ }^{5}$ Department of Pathology, Chang Gung Memorial Hospital and Chang Gung University, \\ Taoyuan, Taiwan; ${ }^{6}$ Department of Medical Oncology, Chang Gung Memorial Hospital and Chang Gung University, Taoyuan, Taiwan; \\ and ${ }^{7}$ Department of Diagnostic Radiology, Chang Gung Memorial Hospital and Chang Gung University, Taoyuan, Taiwan
}

The aim of this single-center study was to investigate whether obtaining an additional PET/CT scan before adjuvant radiotherapy or concurrent chemoradiotherapy (CCRT) could meaningfully improve 2-y disease-free survival (DFS) and disease-specific survival (DSS) rates. Methods: Six hundred seventy-four patients with oral cavity squamous cell carcinoma who received adjuvant therapy after radical surgery were included. Of these, 152 patients were initially scheduled to receive an additional preradiotherapy/CCRT PET/CT scan within $1 \mathrm{wk}$ of starting adjuvant therapy. However, 16 patients were excluded because of either medical problems or refusal. Therefore, 136 patients underwent a preradiotherapy/CCRT PET/ CT scan (PET group), and 522 did not (NO-PET group). All of the participants were followed up for at least $2 \mathrm{y}$ or censored at the last follow-up. The impact of preradiotherapy/CCRT PET/CT imaging was examined using Kaplan-Meier curves and Cox proportional hazards models. Results: Two-year DFS $(80 \%$ vs. $70 \%, P=$ $0.033)$ and DSS $(84 \%$ vs. $75 \%, P=0.010)$ rates were significantly higher in the PET than in the NO-PET group. In the PET group, both DFS and DSS were higher in patients with negative findings than in those without ( $88 \%$ vs. $22 \%$ and $91 \%$ vs. $36 \%$, respectively; both $P$ $<0.001)$. A prognostic scoring system based on the presence of the 2 independent risk factors in the PET group (extracapsular spread and lymphatic invasion) predicted both DFS $(P=0.001$ and $P<$ 0.001 , respectively) and DSS $(P=0.001$ and $P<0.001$, respectively). Nineteen patients (14\%) had their treatment modified by preradiotherapy/CCRT PET/CT findings. Of these, 15 were treated with curative intent due to the presence of locoregional disease, and 4 received palliative care due to distant metastases. Seven of the 15 patients are currently alive without disease. Conclusion: An additional preradiotherapy/CCRT PET/CT scan improves both DFS and DSS in patients with advanced oral cavity squamous cell carcinoma.

\footnotetext{
Received Jul. 7, 2014; revision accepted Nov. 20, 2014.

For correspondence or reprints contact: Tzu-Chen Yen, Department of Nuclear Medicine, Chang Gung Memorial Hospital at Linkou, No. 5, Fu-Hsing ST., Kwei-Shan, Taoyuan, Taiwan.

E-mail: yen1110@adm.cgmh.org.tw

${ }^{*}$ Contributed equally to this work.

tContributed equally to this work.

Published online Dec. 18, 2014.

COPYRIGHT (c) 2015 by the Society of Nuclear Medicine and Molecular Imaging, Inc.
}

Key Words: oral squamous cell carcinoma; FDG PET/CT; adjuvant therapy

J Nucl Med 2015; 56:22-30

DOI: 10.2967/jnumed.114.145300

$\mathbf{O}$ al cavity squamous cell carcinoma (OSCC) represents a growing burden in Southern Asian countries, including Taiwan (1). Despite public health policies aimed at reducing common risky oral habits (e.g., alcohol drinking, betel nut chewing, and cigarette smoking), the incidence of OSCC in the Taiwanese population rose steeply by $30 \%$ in the last $5 \mathrm{y}$. Because of its adverse functional, cosmetic, and prognostic impact, OSCC poses a serious threat to public health and health-care systems and carries heavy personal and societal costs.

Taiwanese OSCC patients are characterized by a high frequency of advanced disease at presentation (52\%) (2). Notably, more than $80 \%$ of all recurrences occur in patients with advanced primary disease, mainly within 2 y of definitive treatment (3-9). The key to decreasing recurrence rates is not only to identify (and control) major risk factors, but also to create evidence-based guidelines for the treatment and follow-up of patients with advanced disease (10-12). Certain primary tumor biomarkers identified in studies focusing on the role of genetic variants or viruses in OSCC have shown potential utility as predictors or prognostic factors. However, results have been frequently controversial, requiring further verification and validation before being integrated into treatment guidelines (13-17). Thus, the major problem in treating OSCC is that prognostic stratification continues to rely on pathologic variables, which are not readily available before radical surgery. In general, patients with advanced disease are treated with adjuvant therapy 6-8 wk after radical surgery $(18,19)$. However, such an approach has significant shortcomings, including immunosuppression and pain directly related to food intake. Notably, we have previously shown that $22 \%$ of OSCC patients with pathologically positive regional lymph nodes have false-negative PET findings during primary staging (20). Ultimately, the evolution of occult metastases to clinically evident disease in the time frame between 
radical surgery and adjuvant therapy would result in treatment failures. Moreover, we previously demonstrated that a short time to recurrence $(<10 \mathrm{mo})$ predicts negative outcomes after salvage attempts in patients with disease recurrence diagnosed on clinical grounds (21). Furthermore, we were able to show that an additional PET/CT scan before radiotherapy or concurrent chemoradiotherapy (CCRT) may help define adjuvant strategies in advanced OSCC patients through the detection of new unexpected lesions after primary staging (10). On the basis of these observations, we hypothesized that an additional preradiotherapy/CCRT PET/CT scan would detect at least half of patients with either rapidly growing regional lymph nodes or distant metastases that were occult at the time of PET imaging performed for primary staging. Of these, at least $12 \%$ may change their adjuvant treatment plan and could achieve at least $9 \%$ long-term survival by having their clinical management modified by the additional preradiotherapy/ CCRT PET findings. Starting from these premises, the aim of the present single-center study was to investigate whether obtaining a PET/CT scan before radiotherapy or CCRT could meaningfully improve ( $\geq 9 \%$ ) disease-free survival (DFS) and disease-specific survival (DSS) rates at $2 \mathrm{y}$. We also aimed to define the priority of preradiotherapy/CCRT PET/CT imaging among OSCC patients.

\section{MATERIALS AND METHODS}

\section{Ethical Statement}

The study protocol complied with the tenets of the Declaration of Helsinki and was approved by the Institutional Review Board of the Chang Gung Memorial Hospital (101-0668C and 102-1577C). All patients provided written informed consent to be included in the prospective study. All of the data were securely protected (by delinking identifying information from the main datasets), and access to the information was made available only to investigators, and analyzed anonymously.

\section{Patients}

We designed the current study as a single-center investigation. Between January 2000 and May 2012, we identified 674 patients with pathologically proven OSCC who were scheduled to receive adjuvant therapy with radiotherapy or CCRT because of the presence of pathologic risk factors. All of the patients had undergone radical surgery with neck dissection and had no evidence of distant metastases on preoperative imaging studies. Before August 2001, MR imaging or CT was used to screen for metastases, whereas MR imaging/CT and PET/CT were used thereafter. As of October 2008, we obtained an additional PET/CT scan within $1 \mathrm{wk}$ of starting adjuvant therapy with radiotherapy or CCRT. For the purpose of the study, all of the patients enrolled before October 2008 served as controls (NO-PET group, $n=$ 522). The remaining 152 patients who were recruited thereafter were deemed eligible as potential index cases (PET group). Of the 152 patients, 16 did not meet the inclusion criteria (11 subjects were unable to receive adjuvant therapies within $6 \mathrm{wk}$ after radical surgery because of poor general conditions, and 5 patients refused to receive a second PET scan). Finally, 136 patients were included in the PET group (Fig. 1). All of the participants provided their written informed consent before inclusion in the study. All of the patients in the 2 groups were uniformly treated according to the current guidelines or by consensus of our oncology team. All patients expressed willingness to undergo a CT- or ultrasound-guided biopsy or surgical exploration, if necessary. Exclusion criteria were as follows: previous diagnosis of another malignancy, refusal or inability to receive definitive treatment, and presence of hyperglycemia (defined as serum glucose levels $>$ $200 \mathrm{mg} / \mathrm{dL}$ ). All participants underwent a thorough presurgical eval-

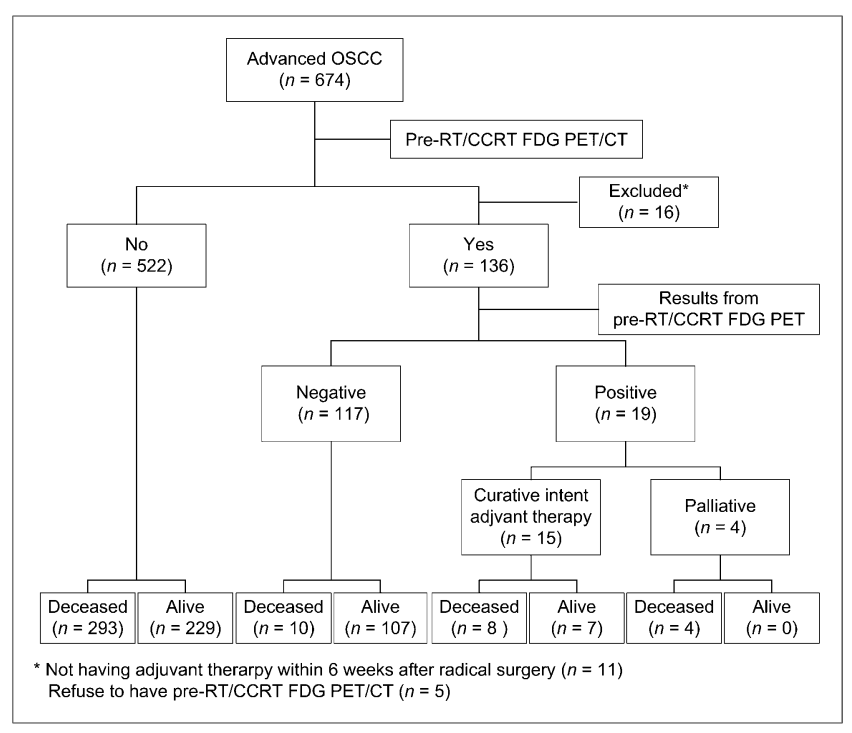

FIGURE 1. Flowchart of study. RT = radiotherapy.

uation in the 2 wk preceding primary surgery as previously described (3). Primary staging was performed in accordance with the criteria from the 2002 American Joint Committee on Cancer (sixth edition) (22).

\section{PET/CT Imaging}

All of the PET/CT scans were obtained using a Discovery ST 16 PET/CT scanner (GE Healthcare) as previously reported in detail (10). We carefully reviewed all PET, CT, and fused PET/CT images as displayed in the axial, coronal, and sagittal planes. We examined both noncorrected and attenuation-corrected PET images, which were also assessed in rotating maximum-intensity projections. Regions of interest were drawn over PET-diagnosed lesions or-in the event of such lesions being absent-using the corresponding CT images. After measuring the highest activity within each region of interest, we calculated the standardized uptake value as the highest activity concentration per injected dose (per body weight in $\mathrm{kg}$ ) after correction for the radioactive decay. Abnormal foci of increased ${ }^{18} \mathrm{~F}$-FDG uptake on PET/CT images were scored as previously described on a 5-point scale (10). In general, positive findings are considered to be present for visual scores of 3 or 4, equivocal results are characterized by a score of 2 , and scores of 0 or 1 denote negative findings. However, because the study specifically focused on additional PET/CT imaging at 5-6 wk after radical surgery, only lesions with a score 4 were considered as positive PET/CT findings. All of the PET/CT images were interpreted by consensus of 2 experienced nuclear medicine physicians and 1 radiologist. The results of histopathology were considered as the gold standard.

\section{Treatment Protocol}

The surgical principles used for the patients enrolled in this study have been previously reported and were based on the following parameters: primary tumor, surgical margin, classic radical or modified neck dissection (level I-V), supraomohyoid neck dissection (levels I-III), and bilateral neck dissection (3). The surgical defects were repaired with primary closure or reconstructed immediately by plastic surgeons using free or local flaps. Postoperative radiotherapy was scheduled within 6-8 wk after radical surgery and administered using a $6 \mathrm{MV}$ x-ray beam produced by a linear accelerator. The total dose, prophylactic radiation dose, boost to the total dose, elective neck irradiation, ipsilateral whole-neck prophylactic irradiation, wholeneck elective irradiation, and radiation fields were determined as previously described (3). CCRT consisted of intravenous cisplatin, 30-40 
TABLE 1

General Characteristics of Study Participants $(n=658)$

\begin{tabular}{|c|c|c|c|c|c|}
\hline Characteristic & $n$ & $\%$ & PET group $(n=136)$ & NO-PET group $(n=522)$ & $P$ \\
\hline Age $(y)$ & & & & & 1.000 \\
\hline$\leq 40$ & 112 & 17 & $23(17)$ & $89(17)$ & \\
\hline$>40$ & 546 & 83 & $113(83)$ & $433(83)$ & \\
\hline Treatment modality & & & & & 1.000 \\
\hline Surgery & 17 & 2 & $3(2)$ & $14(3)$ & \\
\hline Surgery + radiotherapy/CCRT & 641 & 98 & $133(98)$ & $508(97)$ & \\
\hline Pathologic T status & & & & & 0.203 \\
\hline pT1-3 & 394 & 60 & $88(65)$ & $306(59)$ & \\
\hline pT4 & 264 & 40 & $48(35)$ & $216(41)$ & \\
\hline Pathologic $\mathrm{N}$ status & & & & & 0.110 \\
\hline NO & 237 & 36 & $57(42)$ & $180(35)$ & \\
\hline $\mathrm{N}+$ & 421 & 64 & $79(58)$ & $342(65)$ & \\
\hline Pathologic stage & & & & & 0.123 \\
\hline p stage I-III & 214 & 32 & $52(38)$ & $162(31)$ & \\
\hline p stage IV & 444 & 68 & $84(62)$ & $360(69)$ & \\
\hline Tumor depth (mm) & & & & & 0.528 \\
\hline$<10$ & 196 & 30 & $37(27)$ & $159(31)$ & \\
\hline$\geq 10$ & 462 & 70 & $99(73)$ & $363(69)$ & \\
\hline Margin status (mm) & & & & & 0.007 \\
\hline$\leq 4$ & 87 & 13 & $28(21)$ & $59(11)$ & \\
\hline$>4$ & 571 & 87 & $108(79)$ & $463(89)$ & \\
\hline Extracapsular spread & & & & & 0.061 \\
\hline No & 397 & 60 & $92(68)$ & $305(58)$ & \\
\hline Yes & 261 & 40 & $44(32)$ & 217 (42) & \\
\hline Cell differentiation & & & & & 0.194 \\
\hline Well/moderate & 574 & 87 & $114(84)$ & $460(88)$ & \\
\hline Poor & 84 & 13 & $22(16)$ & $62(12)$ & \\
\hline Perineural invasion & & & & & 0.001 \\
\hline No & 342 & 52 & $53(39)$ & $289(55)$ & \\
\hline Yes & 316 & 48 & $83(61)$ & $233(45)$ & \\
\hline Vascular invasion & & & & & 0.018 \\
\hline No & 634 & 96 & $126(93)$ & $508(97)$ & \\
\hline Yes & 24 & 4 & $10(7)$ & $14(3)$ & \\
\hline Lymphatic invasion & & & & & 0.236 \\
\hline No & 607 & 92 & $128(94)$ & $479(92)$ & \\
\hline Yes & 51 & 8 & $8(6)$ & $43(8)$ & \\
\hline Changes in treatment protocol* & & & & & 0.456 \\
\hline No & 580 & 88 & $123(90)$ & 457 (88) & \\
\hline Yes & 78 & 12 & $13(10)$ & $65(12)$ & \\
\hline
\end{tabular}

*Treatment protocol was not followed as originally planned.

Data in parentheses are percentages.

$\mathrm{mg} / \mathrm{m}^{2}$ weekly or $100 \mathrm{mg} / \mathrm{m}^{2}$ every $3 \mathrm{wk}$. Patients in poor general conditions or who refused chemotherapy were treated with postoperative adjuvant radiotherapy alone.

\section{Study Power}

Given an $\alpha$ level of 0.05 and a power of $80 \%$, the sample size was calculated, aiming for a $9 \%$ improvement in the 2 -y DFS from $70 \%$ in patients who did not undergo preradiotherapy/CCRT PET/CT imaging. The calculation deemed that at least 126 patients per study arm were required for the study.

\section{Statistical Analysis}

Follow-up was continued until May 2014 or until death. All of the time intervals were calculated from the date of the primary treatment 


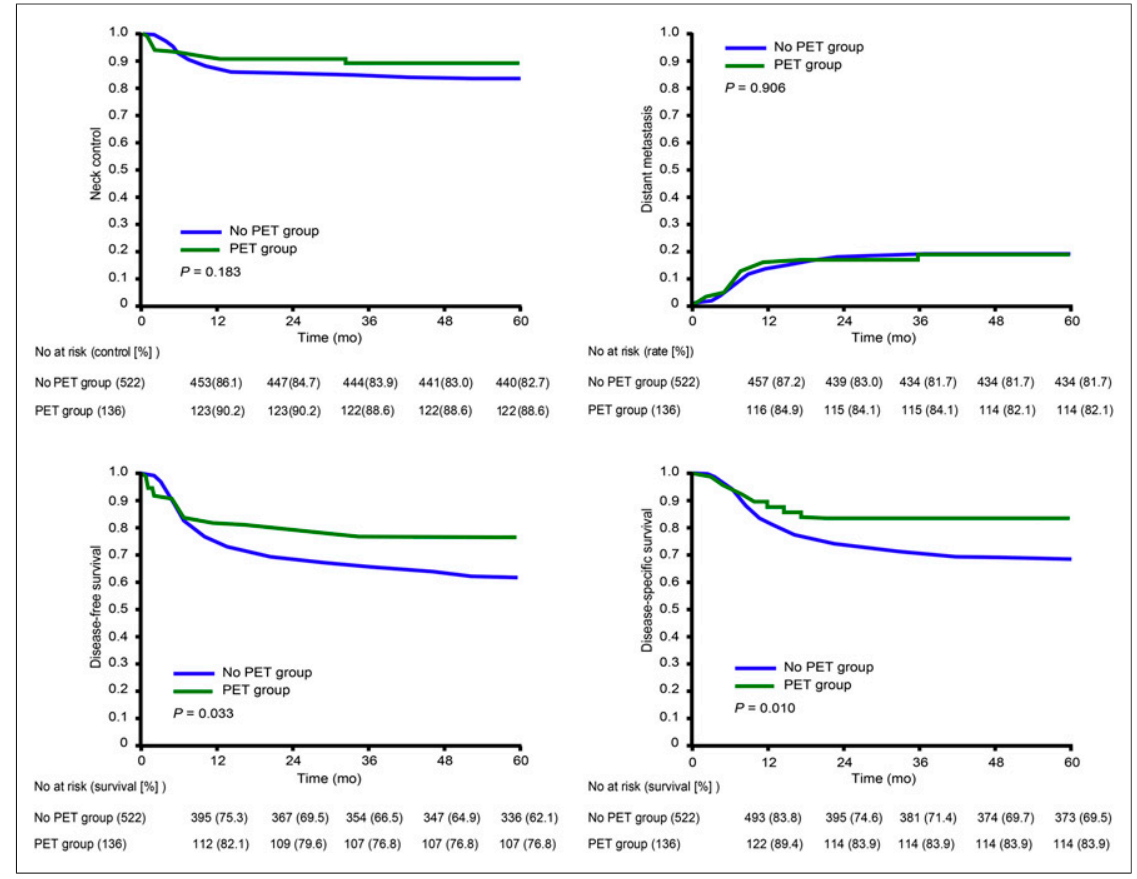

FIGURE 2. Kaplan-Meier curves for different survival endpoints (neck control, distant metastases, DFS, and DSS) in PET and NO-PET groups.

to the event of interest or censored at $2 \mathrm{y}$ for surviving patients. DSS was defined as the survival until death from cancer-related causes. DFS was calculated as the interval until the diagnosis of local recurrence, regional relapse, or distant metastasis. The impact of preradiotherapy/CCRT PET/CT imaging was examined using KaplanMeier curves (log-rank test) and multivariate Cox proportional hazards regression models. To address the potential selection bias due to the lack of randomization, we calculated the propensity score for

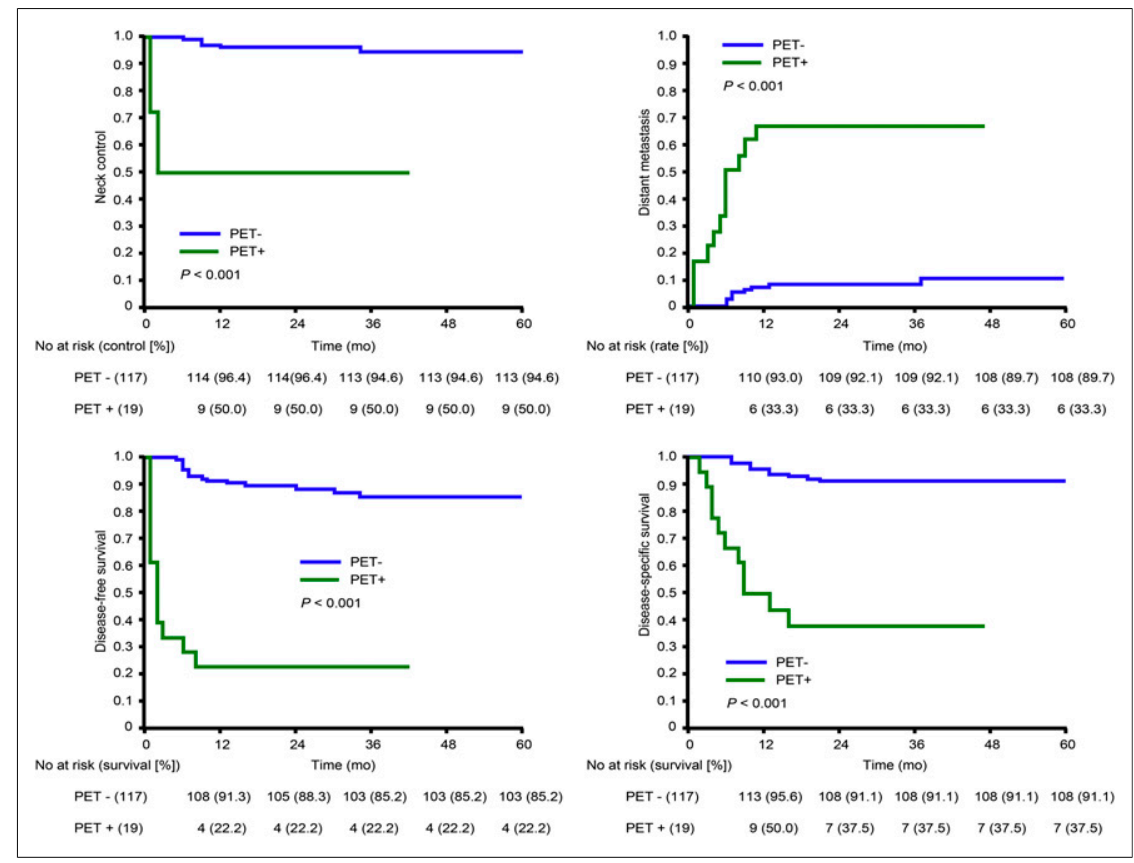

FIGURE 3. Kaplan-Meier curves for different survival endpoints (neck control, distant metastases, DFS, and DSS) according to results (positive vs. negative) of preradiotherapy/CCRT $\mathrm{PET} / \mathrm{CT}$ scan. clinical outcomes (DFS and DSS) in both PET and NO-PET groups according to different confounding factors. The proportional hazard assumptions were tested and found to be valid. All analyses were performed using the SPSS software (version 18.0; SPSS Inc.). Statistical significance was defined as a $P$ value of less than 0.05 (2-tailed).

\section{RESULTS}

The general characteristics of the PET $(n=136)$ and NO-PET $(n=522)$ groups are demonstrated in Table 1 . The 2 groups were found to differ significantly in terms of margin status of $4 \mathrm{~mm}$ or less, perineural invasion, and vascular invasion. The median follow-up time was 29 mo (mean, $29 \mathrm{mo}$; range, 2-60 mo) and $58 \mathrm{mo}$ (mean, $62 \mathrm{mo}$; range, 1-169 mo) in the PET and NO-PET groups, respectively. At the time of analysis, the distribution of events in the PET group was as follows: local recurrence $(n=6 ; 4 \%)$, neck recurrence $(n=14$; $10 \%)$, and distant metastases $(n=22$; $16 \%)$. One hundred fourteen patients $(84 \%)$ were alive. In the NO-PET group, there were 89 patients $(17 \%)$ with local recurrence, $85(16 \%)$ with neck recurrence, and 88 (17\%) with distant metastases. Two hundred twenty-nine patients (44\%) were alive.

\section{Outcomes According to Preradiotherapy/CCRT PET/CT Imaging}

Figure 2 depicts the clinical outcomes in the PET and NO-PET groups. Compared with patients in the NO-PET group, those in the PET group showed significantly higher 2-y DFS ( $80 \%$ vs. $70 \%, P=0.033)$ and DSS ( $84 \%$ vs. $75 \%, P=0.010)$ rates. To address the potential selection bias due to the lack of randomization, the propensity scores for clinical outcomes in both PET and NO-PET groups were determined according to confounding factors found to have $P$ values less than 0.5 in Table 1 . Specifically, the following factors were analyzed: pathologic $\mathrm{T}$ status, pathologic $\mathrm{N}$ status, pathologic stage, margin status, presence of extracapsular spread, cell differentiation, perineural invasion, vascular invasion, and lymphatic invasion. After applying this procedure, we observed a nonsignificant trend for differences between the PET and NOPET groups in terms of DFS (adjusted $P=$ 0.081). Notably, a significant difference was found for DSS (adjusted $P=0.033$ ).

Among patients who underwent preadjuvant PET, those with negative findings had significantly higher neck control $(96 \%$ vs. $50 \%, P<0.001)$, DFS ( $88 \%$ vs. $22 \%$, $P<0.001)$, and DSS (91\% vs. $36 \%, P<$ $0.001)$ rates than those with positive results. Negative PET findings were also as- 

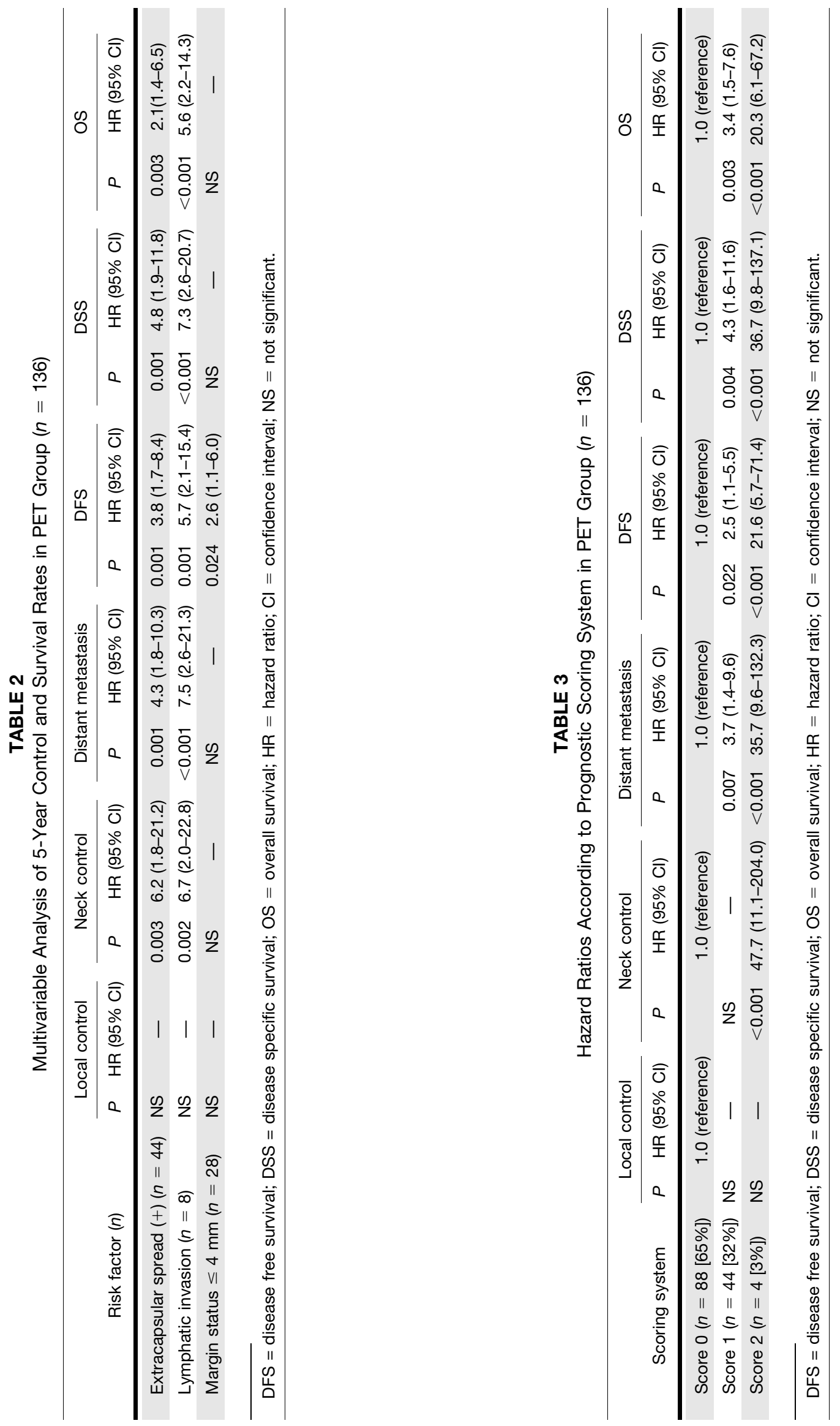


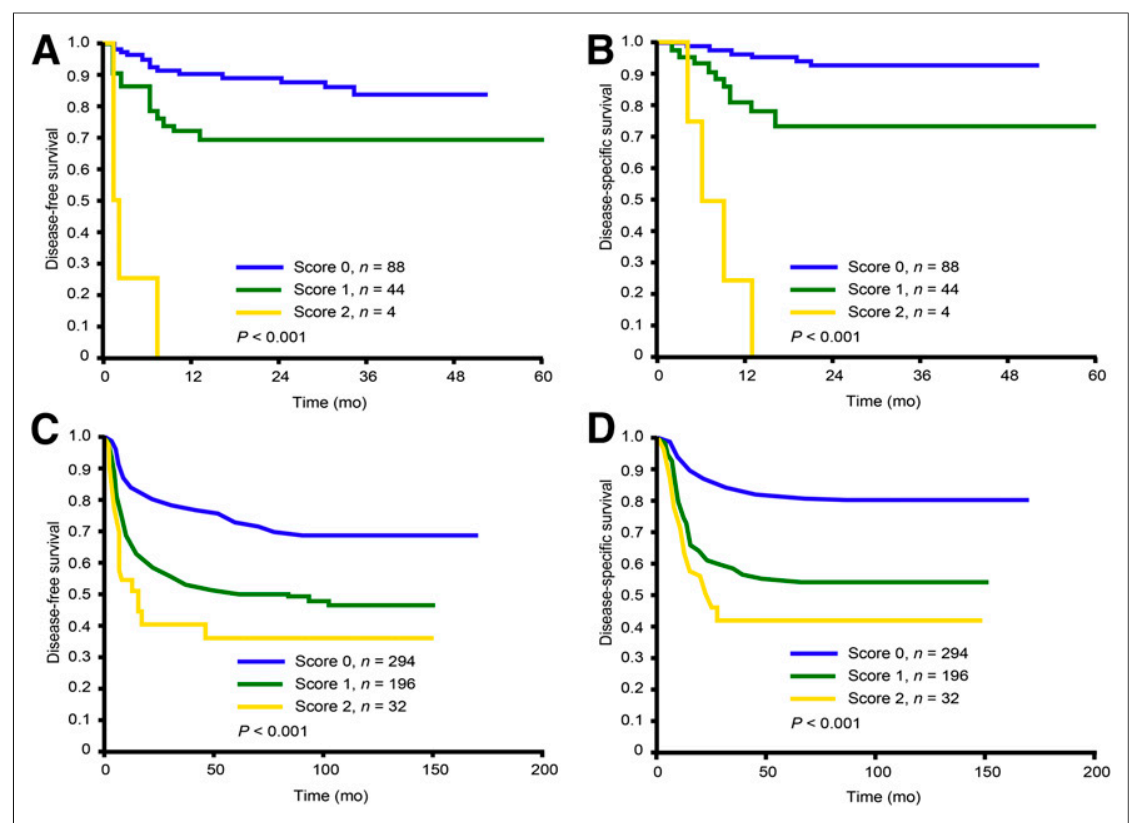

FIGURE 4. Kaplan-Meier curves for different survival endpoints (DFS and DSS) in PET (A and B) and NO-PET (C and D) groups according to prognostic scoring system.
1-2 were more likely to have positive findings than those with a score of 0 (score 1 , odds radio $=4.02, P=0.012$; score 2 , odds ratio $=41.00, P=0.003)$. According to our prognostic scoring system, 6 of the 19 patients with PET true-positive results had a score of 0 (low-risk), 10 had a score of 1 (intermediate-risk), and 3 had a score of 2 (high-risk). In the low-risk group, 3 patients had local disease, 1 had regional disease, 1 had locoregional disease, and the remaining 1 had multiple distant metastases to the lung. The latter patient received supportive treatment and died of disease. The remaining 5 patients were treated with curative-intent CCRT. Three are currently alive without disease, 1 is alive with disease, and 1 died. All of the 7 intermediaterisk patients who were found to have locoregional disease had received CCRT with curative intent. Of these, 3 died of disease and 2 are currently alive without disease. Similarly, all of the 3 patients in the high-risk group showed locoregional disease and received CCRT with curative sociated with a lower rate of distant metastasis $(8 \%$ vs. $67 \%, P<$ 0.001) (Fig. 3).

\section{Independent Risk Factors and Prognostic Scoring System}

We used multivariate analysis to identify the independent predictors of outcomes in the PET group (Table 2). The results indicated that extracapsular spread and lymphatic invasion were independent predictors of neck control rate, distant metastasis rate, DSS, DFS, and overall survival, whereas a margin status of $4 \mathrm{~mm}$ or less was independently associated with DFS alone. We then constructed a 3-point prognostic scoring system by summing up the 2 independent prognostic factors (extracapsular spread and lymphatic invasion) by assigning a score of 0 in the absence and 1 in the presence of each variable. As expected, high-risk patients with a score of 2 had a poor prognosis for all survival endpoints, the only exception being local control (Table 3). Intermediate-risk patients with a score of 1 had a worse prognosis than low-risk patients (who scored 0) in terms of DSS, DFS, overall survival, and distant metastasis rates. Although the prognostic score showed a significant association with both DFS and DSS in the PET group (both $P<0.001$ ), these results were expected because such a scoring system was constructed on the basis of the findings obtained in the 136 patients of the PET group. To validate the usefulness of this score, we tested its prognostic impact in the PET group in terms of DFS and DSS rates. Notably, the score retained its significant prognostic value in the NO-PET group (Fig. 4, PET group, A-B; NO-PET group, C-D; both $P<0.001)$.

\section{Clinical Impact of Preradiotherapy/CCRT PET/CT Imaging}

Preradiotherapy/CCRT PET/CT imaging identified unexpected lesions in 26 patients (Table 4). Nineteen patients showed truepositive PET findings confirmed by either histopathology or imaging follow-up (all of these lesions had a score of 4 on PET/CT scans). In contrast, 7 patients did not have a final confirmation of PET results. In the PET group, patients with a prognostic score of intent. Unfortunately, all of them died within 10 mo of surgery. Among the 7 patients with lack of confirmation, 4 did not have any treatment change (patients 20,21, 22, and 24). Although patient 23 had lesions with a PET score of 3 at level 2 right neck lymph nodes and at a middle right lung lesion, the results of neck lymph node biopsy were negative. Despite a dose change in the right neck region, additional radiotherapy at the lung was not performed and the patient died of respiratory failure due an extensive pleural effusion. In 2 other cases (patients 25 and 26), radiotherapy dose and field were changed despite negative results on neck lymph node biopsies. On the basis of these findings, we concluded that PET yielded false-positive results in patients 20,21, 22, and 24. However, it is difficult to classify PET results as true- or falsepositive in patients 23,25 , and 26 (Table 4). The clinicopathologic characteristics and clinical outcomes of the 15 OSCC patients experiencing DFS events after radical surgery and who showed negative findings on preradiotherapy/CCRT PET/CT are summarized in Supplemental Table 1 (supplemental materials are available at http://jnm.snmjournals.org). The corresponding images are reported in the supplemental materials. Among these 15 patients, 11 died and 4 were alive without evidence of disease. No falsenegative findings were found on preradiotherapy/CCRT PET/CT imaging.

\section{DISCUSSION}

This study is the first, to our knowledge, to describe the impact of preradiotherapy/CCRT PET/CT imaging in a large series of OSCC patients who were scheduled to receive adjuvant therapy after radical surgery. Our present investigation was prompted by previous findings demonstrating that PET/CT is superior to conventional imaging modalities (CT and MR imaging) for primary staging, especially at the neck lymph nodes. Moreover, patients who present with advanced OSCC at diagnosis are generally treated with adjuvant therapy $6-8 \mathrm{wk}$ after radical surgery. Treatment planning in such patients is generally based on the results 
TABLE 4

Clinicopathologic Characteristics and Clinical Outcomes of OSCC Patients Who Had Positive Findings on Preradiotherapy/CCRT PET/CT Imaging

\begin{tabular}{|c|c|c|c|c|c|c|c|c|c|c|}
\hline \multirow[b]{2}{*}{$\begin{array}{l}\text { Case } \\
\text { no. }\end{array}$} & \multirow{2}{*}{$\begin{array}{l}\text { Primary } \\
\text { cancer } \\
\text { site }\end{array}$} & \multirow[b]{2}{*}{$\begin{array}{l}\text { Primary } \\
\text { staging }\end{array}$} & \multicolumn{3}{|c|}{ Prognostic scoring system } & \multirow[b]{2}{*}{$\begin{array}{l}\text { Pre-RT/CCRT } \\
\text { PET findings }\end{array}$} & \multirow[b]{2}{*}{ Evidence } & \multirow{2}{*}{$\begin{array}{c}\text { Adjuvant } \\
\text { treatment } \\
\text { modification }\end{array}$} & \multirow[b]{2}{*}{ Outcomes } & \multirow[b]{2}{*}{$\begin{array}{l}\text { Follow-up } \\
\text { (mo) }\end{array}$} \\
\hline & & & $\begin{array}{l}\text { Extracapsular } \\
\text { spread }\end{array}$ & $\begin{array}{l}\text { Lymphatic } \\
\text { invasion }\end{array}$ & Score & & & & & \\
\hline 1 & Left tongue & pT2NOMO & $\mathrm{N}$ & $\mathrm{N}$ & 0 & Local, LN (left N3) & $\begin{array}{l}\text { Imaging } \\
\text { follow-up }\end{array}$ & $\begin{array}{l}\text { Curative } \\
\text { CCRT }^{\star}\end{array}$ & NED & 49 \\
\hline 2 & Right tongue & pT4aN1M0 & $\mathrm{N}$ & $\mathrm{N}$ & 0 & LN (right, level IV) & Tissue proven & Curative $\mathrm{CCRT}^{\dagger}$ & NED & 24 \\
\hline 3 & Right bucca & pT4aNOMO & $\mathrm{N}$ & $\mathrm{N}$ & 0 & Local & $\begin{array}{l}\text { Imaging } \\
\text { follow-up }\end{array}$ & Curative $\mathrm{CCRT}^{\star}$ & NED & 42 \\
\hline 4 & Left bucca & pT2NOMO & $\mathrm{N}$ & $\mathrm{N}$ & 0 & Lung & $\begin{array}{l}\text { Imaging } \\
\text { follow-up }\end{array}$ & Palliation & DOD & 4 \\
\hline 5 & Left tongue & pT3NOMO & $\mathrm{N}$ & $\mathrm{N}$ & 0 & Local & $\begin{array}{l}\text { Imaging } \\
\text { follow-up }\end{array}$ & Curative $\mathrm{CCRT}^{\star}$ & NED & 34 \\
\hline 6 & Left bucca & pT4aNOMO & $\mathrm{N}$ & $\mathrm{N}$ & 0 & Local & $\begin{array}{l}\text { Imaging } \\
\text { follow-up }\end{array}$ & Curative CCRT* & DOD & 12 \\
\hline 7 & Right tongue & pT4aN2bM0 & $P$ & $\mathrm{~N}$ & 1 & LN (left, level V) & Tissue roven & Curative $\mathrm{CCRT}^{\dagger}$ & NED & 47 \\
\hline 8 & Right bucca & pT2N2bM0 & $P$ & $\mathrm{~N}$ & 1 & Local & $\begin{array}{l}\text { Imaging } \\
\text { follow-up }\end{array}$ & Curative CCRT* & NED & 26 \\
\hline 9 & Right tongue & pT4aN2cM0 & $P$ & $\mathrm{~N}$ & 1 & LN (left, level IV) & $\begin{array}{l}\text { Imaging } \\
\text { Follow-up }\end{array}$ & Curative $\mathrm{CCRT}^{\dagger}$ & DOD & 13 \\
\hline 10 & Right tongue & pT2N2bM0 & $P$ & $\mathrm{~N}$ & 1 & LN (left, level I, II, III) & Tissue proven & Curative $\mathrm{CCRT}^{\dagger}$ & DOD & 16 \\
\hline 11 & Left tongue & pT2N2bM0 & $P$ & $\mathrm{~N}$ & 1 & LN (right, level III, V) & $\begin{array}{l}\text { Imaging } \\
\text { follow-up }\end{array}$ & Curative $\mathrm{CCRT}^{\dagger}$ & NED & 31 \\
\hline 12 & Right tongue & pT2N2cM0 & $P$ & $\mathrm{~N}$ & 1 & LN (right, level III, IV) & Tissue proven & Curative $\mathrm{CCRT}^{\dagger}$ & DOD & 9 \\
\hline 13 & Right bucca & pT4aN2bM0 & $P$ & $\mathrm{~N}$ & 1 & Lung & $\begin{array}{l}\text { Imaging } \\
\text { follow-up }\end{array}$ & Palliation & DOD & 2 \\
\hline 14 & Left bucca & pT3N2bM0 & $P$ & $\mathrm{~N}$ & 1 & $\begin{array}{l}\text { Local, LN (left, level } \\
\text { III), lung }\end{array}$ & $\begin{array}{l}\text { Tissue } \\
\text { proven }\end{array}$ & Palliation & DOD & 5 \\
\hline 15 & $\begin{array}{l}\text { Left mouth } \\
\text { floor }\end{array}$ & pT4aN2bM0 & $P$ & $\mathrm{~N}$ & 1 & Lung & $\begin{array}{l}\text { Imaging } \\
\text { follow-up }\end{array}$ & Palliation & DOD & 3 \\
\hline 16 & $\begin{array}{l}\text { Right alveolar } \\
\text { ridge }\end{array}$ & pT4aN1M0 & $\mathrm{P}$ & $\mathrm{N}$ & 1 & Local & $\begin{array}{l}\text { Imaging } \\
\text { follow-up }\end{array}$ & Curative CCRT* & DOD & 8 \\
\hline 17 & Right bucca & pT4aN2bM0 & $P$ & $P$ & 2 & $\begin{array}{l}\text { Local, LN (left, level } \\
\text { V and parotid) }\end{array}$ & Tissue proven & Curative $\mathrm{CCRT}^{\dagger}$ & DOD & 4 \\
\hline 18 & Right bucca & pT4aN2bM0 & $P$ & $\mathrm{P}$ & 2 & $\begin{array}{l}\text { LN (left, level IV and } \\
\text { parotid) }\end{array}$ & $\begin{array}{l}\text { Imaging } \\
\text { follow-up }\end{array}$ & Curative $\mathrm{CCRT}^{\ddagger}$ & DOD & 9 \\
\hline 19 & $\begin{array}{l}\text { Right alveolar } \\
\text { ridge }\end{array}$ & pT3N2bM0 & $P$ & $\mathrm{P}$ & 2 & $\begin{array}{l}\text { LN (right, level V } \\
\text { and left, parotid) }\end{array}$ & Tissue proven & Curative $\mathrm{CCRT}^{\dagger}$ & DOD & 6 \\
\hline 20 & $\begin{array}{l}\text { Left } \\
\text { retromolar } \\
\text { trigone }\end{array}$ & pT4aNOMO & $\mathrm{N}$ & $\mathrm{N}$ & 0 & Lung score $3^{\S}$ & $\begin{array}{l}\text { Imaging } \\
\text { follow-up }\end{array}$ & Radiotherapy & NED & 36 \\
\hline 21 & Right buccal & pT4aNOMO & $\mathrm{N}$ & $\mathrm{N}$ & 0 & Lung score $3^{\S}$ & $\begin{array}{l}\text { Imaging } \\
\text { follow-up }\end{array}$ & Radiotherapy & NED & 33 \\
\hline 22 & $\begin{array}{l}\text { Right } \\
\text { retromolar } \\
\text { trigone }\end{array}$ & pT2NOMO & $\mathrm{N}$ & $\mathrm{N}$ & 0 & $\begin{array}{l}\text { Local (right mandible, } \\
\text { score } 3^{\S} \text { ) }\end{array}$ & $\begin{array}{l}\text { Tissue-proven } \\
\text { negative }\end{array}$ & Radiotherapy & NED & 60 \\
\hline 23 & Left tongue & pT2N2bM0 & $\mathrm{N}$ & $P$ & 1 & $\begin{array}{l}\text { LN (right, level II, } \\
\text { score } 3^{\S} \text { ), lung, } \\
\text { score } 3^{\S}\end{array}$ & $\begin{array}{l}\text { Tissue- } \\
\text { proven (LN) } \\
\text { negative }\end{array}$ & Curative $\mathrm{CCRT}^{\dagger}$ & $\begin{array}{l}\text { DOD } \\
\text { (pleural } \\
\text { effusion) }\end{array}$ & 8 \\
\hline 24 & $\begin{array}{l}\text { Left alveolar } \\
\quad \text { ridge }\end{array}$ & pT4aNOMO & $\mathrm{N}$ & $\mathrm{N}$ & 0 & $\begin{array}{l}\text { Local (left upper gum, } \\
\text { score } 3^{\S} \text { ) }\end{array}$ & $\begin{array}{l}\text { Tissue-proven } \\
\text { negative }\end{array}$ & Radiotherapy & NED & 33 \\
\hline 25 & Left tongue & pT2N2bM0 & $P$ & $\mathrm{~N}$ & 1 & $\begin{array}{l}\text { LN (right level III, V, } \\
\text { score } 3^{\S} \text { ) }\end{array}$ & $\begin{array}{l}\text { Tissue-proven } \\
\text { negative }\end{array}$ & Curative $\mathrm{CCRT}^{\dagger}$ & NED & 32 \\
\hline 26 & $\begin{array}{l}\text { Right alveolar } \\
\text { ridge }\end{array}$ & pT4aN1M0 & $\mathrm{N}$ & $\mathrm{N}$ & 0 & $\begin{array}{l}\text { LN (right, level V, left } \\
\text { SCF, both scores } 3^{\S} \text { ) }\end{array}$ & $\begin{array}{l}\text { Tissue-proven } \\
\text { negative }\end{array}$ & Curative $\mathrm{CCRT}^{\dagger}$ & NED & 28 \\
\hline
\end{tabular}

${ }^{*}$ Change RT dose; †Change RT dose and field; ${ }^{\ddagger}$ Change field; ${ }^{\S}$ PET/CT score.

$\mathrm{ES}=$ extracapsular spread; $\mathrm{LI}=$ lymphatic invasion; $\mathrm{RT}=$ radiotherapy; $\mathrm{N}=$ negative; $\mathrm{LN}=$ lymph node; $\mathrm{P}=\mathrm{positive;} \mathrm{NED}=$ no evidence of disease, $\mathrm{DOD}$ $=$ died of disease; SCF = supraclavicular fossa. 
of primary staging before surgery and pathology findings after radical dissection. However, some patients may be understaged during primary staging procedures, mostly because of the potential presence of undetectable micrometastases to lymph nodes or distant organs (19). Notably, pain related to radical surgery and reconstructive operations, depressive symptoms, and poor appetite with reduced food intake are excruciating problems in the period between surgery and adjuvant therapy. In this scenario, the growth of occult metastases to clinically evident disease during this time frame would ultimately result in treatment failure. On the basis of our preliminary study (10), an additional preradiotherapy/CCRT PET/CT scan may increase the likelihood of diagnosing previously undetected lesions. We believe that such an advantage might result in significant clinical management changes and improved outcomes in advanced OSCC patients.

Herein, we demonstrated that 2-y DFS and DSS rates were significantly higher in the PET than in the NO-PET group $(10 \%$ and $9 \%$, respectively). In this study, 19 patients (14\%) were found to have early recurrent lesions and had their clinical management modified by preradiotherapy/CCRT PET/CT findings (Table 4). Of these, 15 patients were treated with curative intent after modification of treatment field and dosage, whereas adjuvant treatment was changed to palliation in 4 patients. After a follow-up period ranging from 24 to 49 mo, 7 of the 15 patients who received curative-intent treatment were diseasefree. Notably, 37\% (7/19) of the patients with positive PET results detected within 2 mo of radical surgery would be cured by prompt salvage treatment, suggesting that a short time to recurrence may not be necessarily associated with negative outcomes. Without the additional preradiotherapy/CCRT PET/CT scan, patients with early recurrence would have experienced not only a delayed diagnosis but also a significant undertreatment, ultimately diminishing their likelihood of successful salvage (21). Taken together, these findings indicate that a preradiotherapy/CCRT PET/CT scan may improve the clinical management of OSCC patients by avoiding unnecessary curative attempts (associated with futile patient suffering and inefficient health-care expenditures) and improving survival rates through modifications of adjuvant strategies.

Because preradiotherapy/CCRT PET/CT imaging may significantly increase health-care costs, it is important to define its priority based on the presence of a specific risk factor profile. To this aim, we devised a prognostic scoring system based on the 2 independent risk factors (extracapsular spread and lymphatic invasion) identified in multivariate analysis. We suggest that a preradiotherapy/CCRT PET/CT scan may be justified for all of the intermediate- or high-risk patients (with a score of 1 or 2). Accordingly, these patients showed a reasonably high likelihood of positive findings, being $23 \%$ (10/44) in patients with a score 1 and $75 \%(3 / 4)$ in those with a score 2. Notably, preradiotherapy/ CCRT PET/CT imaging also demonstrated prognostic significance in high-risk patients with a score of 2 . All of the 3 high-risk patients with positive PET/CT results died of disease within 10 mo after surgery because of multiple distant metastases. Further research is needed to establish whether adjuvant therapy with curative intent should be avoided in such patients. Although a cost-effectiveness analysis is needed before making final recommendations, it is important to emphasize that negative results on preradiotherapy/CCRT PET/CT imaging were associated with better clinical outcomes.
Some limitations apply to the reported findings. Because the sample studied consisted of Taiwanese patients enrolled in a betel-quid-chewing endemic area, the results might not apply to other populations that did not have the oral habit of betel quid chewing. Second, the study was not randomized, and potentially more patients with an unfavorable risk profile may have been chosen for preradiotherapy/CCRT PET/CT imaging (Table 1). However, this potential selection bias would result in an attenuation of the positive clinical impact of an additional PET scan, which was not eventually observed in our study. Well-conducted randomized multicenter trials are eagerly awaited to confirm and expand our findings. Finally, external validation of our prognostic score across broader patient populations is needed.

\section{CONCLUSION}

We observed that OSCC patients who underwent preradiotherapy/CCRT PET/CT exhibited improved DFS (by 10\%) and DSS (by 9\%) when compared with patients who did not, underscoring the value of this approach for implementing changes in management and improving survival. We are unaware of the integration of PET data into treatment planning in the National Comprehensive Cancer Network clinical practice guideline of head and neck cancer. An additional preradiotherapy/CCRT PET/CT scan may be justified for patients with advanced OSCC who present with extracapsular spread or lymphatic invasion.

\section{DISCLOSURE}

The costs of publication of this article were defrayed in part by the payment of page charges. Therefore, and solely to indicate this fact, this article is hereby marked "advertisement" in accordance with 18 USC section 1734. This work was financially supported by the Ministry of Science and Technology, Taiwan (grant NMRPG3B6311-3B6313) and the Chang Gung Memorial Hospital at Linkou (grants CMRPG370061-370063, CMRPG3B0021-3B0023, and CMRPG371501-371503). No other potential conflict of interest relevant to this article was reported.

\section{REFERENCES}

1. Scully C, Bagan J. Oral squamous cell carcinoma overview. Oral Oncol. 2009;45:301-308.

2. Cancer registry annual report, Taiwan, 2011. http://tcr.cph.ntu.edu.tw/main.php? Page=N2. Accessed December 10, 2014.

3. Liao CT, Chang JT, Wang HM, et al. Analysis of risk factors of predictive local tumor control in oral cavity cancer. Ann Surg Oncol. 2008;15: 915-922.

4. Sparano A, Weinstein G, Chalian A, Yodul M, Weber R. Multivariate predictors of occult neck metastasis in early oral tongue cancer. Otolaryngol Head Neck Surg. 2004;131:472-476.

5. Liao CT, Chang JT, Wang HM, et al. Survival in squamous cell carcinoma of the oral cavity: differences between pT4 N0 and other stage IVA categories. Cancer. 2007;110:564-571.

6. Liao CT, Wang HM, Ng SH, et al. Good tumor control and survivals of squamous cell carcinoma of buccal mucosa treated with radical surgery with or without neck dissection in Taiwan. Oral Oncol. 2006;42:800-809.

7. Montebugnoli L, Gissi DB, Flamminio F, et al. Clinicopathologic parameters related to recurrence and locoregional metastasis in 180 oral squamous cell carcinomas. Int J Surg Pathol. 2014;22:55-62.

8. Chen TC, Wang CP, Ko JY, et al. The impact of perineural invasion and/or lymphovascular invasion on the survival of early-stage oral squamous cell carcinoma patients. Ann Surg Oncol. 2013;20:2388-2395. 
9. Yanamoto S, Yamada S, Takahashi H, et al. Clinicopathological risk factors for local recurrence in oral squamous cell carcinoma. Int J Oral Maxillofac Surg. 2012;41:1195-1200.

10. Liao CT, Fan KH, Lin CY, et al. Impact of a second FDG PET scan before adjuvant therapy for the early detection of residual/relapsing tumours in highrisk patients with oral cavity cancer and pathological extracapsular spread. Eur J Nucl Med Mol Imaging. 2012;39:944-955.

11. Liao CT, Huang SF, Chen IH, et al. Risk stratification of patients with oral cavity squamous cell carcinoma and contralateral neck recurrence following radical surgery. Ann Surg Oncol. 2009;16:159-170.

12. Liao CT, Wang HM, Chang JT, et al. Analysis of risk factors for distant metastases in squamous cell carcinoma of the oral cavity. Cancer. 2007; 110:1501-1508.

13. Lee LA, Huang CG, Liao CT, et al. Human papillomavirus-16 infection in advanced oral cavity cancer patients is related to an increased risk of distant metastases and poor survival. PLOS ONE. 2012;7:e40767.

14. Olthof NC, Speel EJ, Kolligs J, et al. Comprehensive analysis of HPV16 integration in OSCC reveals no significant impact of physical status on viral oncogene and virally disrupted human gene expression. PLOS ONE. 2014;9: e88718.

15. Onken MD, Winkler AE, Kanchi KL, et al. A surprising cross-species conservation in the genomic landscape of mouse and human oral cancer identifies a transcriptional signature predicting metastatic disease. Clin Cancer Res. 2014; 20:2873-2884.

16. Yoshida R, Nagata M, Nakayama $H$, et al. The pathological significance of Notch1 in oral squamous cell carcinoma. Lab Invest. 2013;93:1068-1081.

17. Hedback N, Jensen DH, Specht L, et al. MiR-21 Expression in the tumor stroma of oral squamous cell carcinoma: an independent biomarker of disease free survival. PLoS ONE. 2014;9:e95193.

18. Wang HM, Wang CS, Chen JS, Chen IH, Liao CT, Chang TC. Cisplatin, tegafur, and leucovorin: a moderately effective and minimally toxic outpatient neoadjuvant chemotherapy for locally advanced squamous cell carcinoma of the head and neck. Cancer. 2002;94:2989-2995.

19. Langendijk JA, de Jong MA, Leemans CR, et al. Postoperative radiotherapy in squamous cell carcinoma of the oral cavity: the importance of the overall treatment time. Int J Radiat Oncol Biol Phys. 2003;57:693-700.

20. Liao CT, Wang HM, Huang SF, et al. PET and PET/CT of the neck lymph nodes improves risk prediction in patients with squamous cell carcinoma of the oral cavity. J Nucl Med. 2011;52:180-187.

21. Liao CT, Chang JT, Wang HM, et al. Salvage therapy in relapsed squamous cell carcinoma of the oral cavity: how and when? Cancer. 2008;112:94-103.

22. Greene F, Balch C, Haller D, et al., ed. American Joint Committee on Cancer. AJCC Cancer Staging Manual. 6th ed. Philadelphia, PA: LippincottRaven; 2002. 